\title{
Public Health Unit notifications of hepatitis C and their follow-up in South Western Sydney,
} Australia

\author{
Elizabeth R O'Brien" ${ }^{a}$, Michelle C Whelana, ${ }^{a}$, Tenzin Lamab and \\ Miriam Levy b,c,d \\ a Department of Gastroenterology, Campbelltown Hospital, Sydney, NSW, Australia \\ b Department of Gastroenterology and Hepatology, Liverpool Hospital, Sydney, NSW, Australia \\ c South Western Sydney Clinical School, UNSW Sydney, Australia \\ d Corresponding author: miriam.levy@health.nsw.gov.au
}

\section{Article history}

Publication date: September 2021 Citation: O'Brien ER, Whelan MC, Lama T, Levy M. Public Health Unit notifications of hepatitis $C$ and their follow-up in South Western Sydney, Australia. First published 17 September 2020. Public Health Res Pract. 2021;31(3):e30342010. https://doi.org/10.17061/phrp30342010

\section{Key points}

- Less than half of patients newly diagnosed with hepatitis $\mathrm{C}$ virus (HCV) in South Western Sydney, Australia, who are eligible for antiviral treatment received such treatment in the 12 months after diagnosis

- Most general practitioners referred new patients with $\mathrm{HCV}$ to specialists, rather than starting patients on treatment themselves. A significant portion of HCV notifications did not receive adequate follow-up

- New Public Health Unit notifications of HCV represent a clear target for investment, to improve continuity of care in the quest towards HCV elimination

\section{Abstract}

Objective: To determine hepatitis $\mathrm{C}$ virus ( $\mathrm{HCV}$ ) treatment rates among those newly diagnosed with the virus in the South Western Sydney Local Health District (SWSLHD) in NSW, Australia.

Study type: Cross-sectional study of patients newly diagnosed with HCV in SWSLHD, based on local Public Health Unit notification data from the second half of 2017.

Methods: A total of 200 consecutive notifications were enrolled in the study. Either the ordering clinician was interviewed, and/or data linkage with local hospital records performed, to determine rates of antiviral treatment in this cohort. Outcomes measured included the proportion of patients: started on antiviral treatment; referred to specialists for consideration of treatment; HCV ribonucleic acid (RNA) negative; and lost to follow-up. Descriptive analysis of factors contributing to those lost to follow-up was performed where available.

Results: The follow-up outcome of $93 \%$ of patients was traced. General Practitioners (GPs) diagnosed a similar number (102) of new HCV cases to those diagnosed by specialists (94). After detecting a patient as HCV antibody positive and confirming active infection, GPs preferred to refer patients to specialists (53\%), rather than further evaluate and treat patients themselves (5\%). The remainder of cases from the GP-detected group were lost to follow-up (26\%), or HCV RNA negative (16\%). Among the speciliastdetected patients, $41 \%$ were treated, $18 \%$ were lost to follow-up, $20 \%$ were RNA negative and the remainder were not treated for reasons including a concurrent diagnosis of hepatocellular carcinoma, or death. The most common reason patients were not started on antiviral treatment was loss to follow-up.

Conclusion: We found that less than half (47\%) of people in South Western Sydney newly diagnosed with HCV, in whom treatment was indicated, received antiviral medication in the 12 months following diagnosis. This figure 
excludes the $25 \%$ cases referred from general practice to specialists, in whom the treatment status is unknown. Approximately one in five newly diagnosed patients (22\%) were lost to follow-up and 18\% were RNA negative, indicating they had no active HCV infection.

\section{Introduction}

Hepatitis C virus ( $\mathrm{HCV}$ ) is a common blood-borne virus, with an Australian population prevalence of approximately $1 \% .{ }^{1}$ Higher prevalence rates are observed in communities of people who inject drugs and migrants ${ }^{2,3}$, which are groups that face barriers to seeking pre-emptive and follow-up medical care. HCV has few symptoms and is not routinely screened for by general practitioners (GPs), so while Australia is now considered a world leader in $\mathrm{HCV}$ treatment ${ }^{4}$, there are many in our community who remain undiagnosed and untreated.

HCV was first listed as a notifiable infection in 1991, shortly after serological testing for the virus became available in Australia. Since this time, Australian laboratories have reported positive anti-HCV antibody test results to their local Public Health Unit (PHU). Notified cases are determined after removing duplicates comprising those who have previously tested positive. ${ }^{5}$ The combined de-identified information has been used to guide resource allocation in blood-borne virus control; PHU notification results have not been used to link individuals to their local clinical services.

The follow-up of a positive antibody test result varies according to the practice of the clinician who ordered the test. Ideally, a patient would follow up with the ordering practitioner and then be tested for HCV ribonucleic acid (RNA) to determine whether their infection was active and, if so, be started on antiviral treatment. Up to $25 \%$ of HCV antibody-positive patients will spontaneously clear the virus within the first 6 months of $\mathrm{HCV}$ infection and become RNA negative ${ }^{6}$, with the remainder developing chronic viraemia (i.e. persisting infection), and becoming potential beneficiaries of antiviral treatment. Patients who remain RNA positive over time face an increased risk of progressive liver fibrosis, which can lead to cirrhosis, liver failure and hepatocellular carcinoma. ${ }^{7}$

The listing of highly efficacious and well-tolerated direct-acting antiviral (DAA) therapies for HCV on Australia's Pharmaceutical Benefits Scheme (PBS) in 2016 enabled population-wide, affordable access to these antiviral therapies, including among those who were incarcerated or reinfected. ${ }^{4}$ The end of interferon-based therapy has reduced the complexity of HCV treatment, enabling increased provision of treatment in primary care. DAA therapies clear the virus in about $95 \%$ of cases following 8-24 weeks of treatment. ${ }^{8}$ An estimated 70260 individuals started DAA therapy in the first 3 years following the PBS listing ${ }^{4}$ of these medications in 2016, representing up to $31 \%$ of patients living with chronic
$\mathrm{HCV}$; with the majority of medications prescribed by nonGP specialists. ${ }^{9}$

At an individual level, the impact of achieving sustained virologic response following DAA therapy reduces a patient's likelihood of developing cirrhosis, hepatocellular carcinoma and liver failure. ${ }^{7}$ Indeed, the number of $\mathrm{HCV}$-positive patients requiring liver transplant referral has already reduced. ${ }^{10}$ Meanwhile, on a population level, successful treatment of a significant portion of patients living with HCV has reduced the population prevalence of $\mathrm{HCV}$, and has also led to a reduction in the incidence rate of $\mathrm{HCV}$ in Australia. ${ }^{4}$

Although the initial uptake of DAA therapy was rapid, it has already started to wane, likely reflecting a 'warehouse effect' where initial treatment numbers reflected a cohort of patients already known to existing clinical services who were unable to achieve viral eradication with previous generations of antiviral therapy. ${ }^{11}$ Reaching the World Health Organization's 2030 target of HCV elimination requires a coordinated, multifaceted approach focusing on primary prevention, increased awareness of $\mathrm{HCV}$, and case-finding with increased testing and treatment. 12,13

Significant investment has been made to educate and support Australian GPs to treat HCV. A number of resources have been developed to facilitate communitybased treatment, for example Project Echo, which is a telementoring, group video conferencing tool used by Liverpool Hospital to link specialist hubs and primary care providers ${ }^{14}$; however much more could be done to maintain the high rate of DAA uptake in Australia.

Within NSW's 15 local health districts, South Western Sydney Local Health District (SWSLHD) is one of the most populous and diverse. In this district, the $\mathrm{PHU}$ receives approximately 370 new HCV notifications per year. ${ }^{15}$ This study aimed to review the proportion of patients recently detected to be HCV-antibody positive in SWSLHD who accessed antiviral treatment, in order to determine whether it would be beneficial to direct resources to this group as part of efforts towards HCV elimination.

\section{Methods}

We obtained PHU notification data on 200 consecutive patients who tested positive for HCV antibody in the study period from 1 August 2017 to 31 January 2018. Cases which had previously tested positive for HCV were removed by standard PHU methodology before the acquisition of the dataset. All notifications were entered into a secure, stand-alone database. 
We established the speciality of the ordering clinician from pathology records for each case and grouped them into three categories: GP, specialist or unknown. We then attempted to determine the diagnosing clinician's contact details. Qualitative and quantitative data were collected via a short, semistructured phone or email questionnaire with the clinician. If telephone interview was not possible, the clinician was directly contacted via email. GP data was collected between January and March 2018, and data from specialists between July and August 2018. The information requested included if the clinician was aware of the critical test result for this patient, if they had arranged an HCV RNA test, whether they had treated the patient for $\mathrm{HCV}$, and if so, whether this was with specialist support (e.g. from Project Echo) or, alternatively, if they had referred the patient on to a specialist, or if the patient was lost to follow-up.

Where information gaps existed (e.g. the ordering clinician was not contactable), data linkage was used: hospital-based electronic medical records for nonadmitted patients were accessed to collect laboratory results and determine treatment status where available. In this way, we sought to fill in gaps by including details of those in the cohort known to one of the local public hospital liver clinics.

The analysis of this data was predominantly qualitative. Interviews were transcribed verbatim. Study results were analysed, summarising key themes, including the relative frequency of GPs' HCV diagnoses compared with those of specialists, the proportion of GPs who directly prescribed antiviral treatment to patients with $\mathrm{HCV}$ and the proportion referring patients for specialist review, and the extent to which patients were lost to follow-up.

The data for this cohort was obtained with permission of the data custodian, the Director of the PHU, SWSLHD. Ethics approval was granted by SWSLHD's Low and Negligible Risk Human Research and Ethics Committee (HE18/171).

\section{Results}

Of the 200 notifications, 102 (51\%) resulted from HCV tests ordered by GPs and 94 (47\%) by non-GP specialists. The speciality status of the ordering clinician for four cases (2\%) could not be determined due to insufficient information on the pathology request form. The outcome of 186 (95\%) of the remaining 196 notifications was determined via phone interview, email questionnaire or data linkage. Due to investigator time limitations, the ordering clinicians for the majority $(81 / 92 ; 88 \%)$ of specialist-diagnosed cases were contacted via email while data linkage was used to determine outcomes for the remaining 11 cases. Among GP-diagnosed cases, $69 \%$ (65/94) of ordering GPs were contacted by phone interview and information for the remaining 29 was obtained by data linkage.
Table 1. Outcomes among patients newly diagnosed with hepatitis $\mathrm{C}$ virus (HCV), by ordering clinician role

\begin{tabular}{|c|c|c|c|}
\hline Outcome & $\begin{array}{c}\text { GP- } \\
\text { diagnosed } \\
\text { cases }\end{array}$ & $\begin{array}{c}\text { Specialist- } \\
\text { diagnosed } \\
\text { cases }\end{array}$ & Total \\
\hline Information available & 94 & 92 & 186 \\
\hline $\begin{array}{l}\text { DAA treatment not } \\
\text { indicated }\end{array}$ & 15 & 30 & 45 \\
\hline HCV RNA-negative ${ }^{a}$ & 15 & 18 & 33 \\
\hline Anti-HCV negativeb & 0 & 3 & 3 \\
\hline Deceased & 0 & 5 & 5 \\
\hline HCC diagnosis & 0 & 4 & 4 \\
\hline $\begin{array}{l}\text { DAA treatment } \\
\text { indicated }\end{array}$ & 79 & 62 & 141 \\
\hline Lost to follow-up & 24 & 17 & 41 \\
\hline $\begin{array}{l}\text { In custody or } \\
\text { incarcerated }\end{array}$ & 0 & 5 & 5 \\
\hline $\begin{array}{l}\text { Patient declined } \\
\text { treatment }\end{array}$ & 0 & 2 & 2 \\
\hline Patients treated & $5(6.3 \%)$ & $38(61 \%)$ & $43(30 \%)$ \\
\hline Referred & $50(63 \%)$ & na & na \\
\hline Treated or referred & $55(70 \%)$ & $38(61 \%)$ & $93(66 \%)$ \\
\hline
\end{tabular}

$\mathrm{GP}=$ general practitioner; $\mathrm{DAA}=$ direct-acting antiviral;

$\mathrm{HCC}=$ hepatocellular carcinoma; $\mathrm{HCV}=$ hepatitis $\mathrm{C}$ virus; na = not applicable

a HCV RNA-negative result indicates no active HCV infection

b Anti-HCV negative result indicates no HCV infection

\section{Patients diagnosed with hepatitis $\mathrm{C}$ virus by general practitioners}

A total of 97 GPs were responsible for 102 notifications. The outcome of 94 of 102 (92\%) GP-identified cases was successfully determined via GP interview or data linkage; 8 were unaccounted for likely due to doctor-related factors (including moving practice location, or taking extended leave). Fifteen were RNA negative, suggesting spontaneous clearance of the virus. The majority (50, $63 \%$ ) of the 79 patients eligible for DAA treatment were referred directly to specialists; the rationale for not treating, as described in interviews, included being too busy and lacking in either confidence or interest in prescribing. Five patients (6\%) were treated by their GP, so a total of 55 patients (70\%) were either treated or referred. A significant number of patients (24, 30\%) were lost to follow-up.

\section{Patients diagnosed with hepatitis C virus by specialists}

Of the 200 notifications, 94 (47\%) were from specialists. Fifty specialists were responsible for a single notification, 
while the remaining 12 specialists were responsible for 44 notifications. The outcome of 92 of 94 specialistidentified cases was successfully determined via interview, email questionnaire or data linkage, with two cases unaccounted for due to non-response. Of the 92 cases, 30 were not eligible for treatment including 18 (20\%) that were RNA negative; 5 (5.5\%) that were deceased; 4 (4.3\%) that were diagnosed with hepatocellular carcinoma; and 3 (3.3\%) that were falsepositive listings (i.e. repeat HCV antibody and RNA negative). Of the 62 cases eligible for treatment, 38 $(61 \%)$ were treated with antiviral therapy. The remaining cases were lost to follow-up, inaccessible due to being in custody or incarcerated, or declined therapy due to moving away from the area.

\section{Discussion}

This study calculated the real-world antiviral treatment rate in those newly diagnosed as HCV-antibody positive. We found that less than half $(47 \%)$ of treatmenteligible new HCV patients in SWSLHD were treated in the 12 months following diagnosis (excluding those referred by GPs to specialists). These findings highlight the significant gap that must be closed to reach $\mathrm{HCV}$ elimination in Australia.

We found that the main reason patients were not treated was a lack of follow-up with their clinician. Almost one-third of patients in this study (41 patients; 29\%) were lost to follow-up, which is cause for concern. It is likely that both patient and system factors play a role. Given HCV infection is usually asymptomatic ${ }^{16}$, many patients may be unaware of the risk posed by persisting viraemia. System factors include the marginalisation and stigma faced by people who inject drugs who are at an increased risk of $\mathrm{HCV}$ infection, which limits their ready access to services ${ }^{17}$, a lack of coordination of patient care, and potentially the low rates of community-based treatment. ${ }^{18}$ Meanwhile, approximately one-quarter of patients in this study were referred from GPs to specialists for consideration of treatment, and while the outcome of this subgroup was unknown, it is likely that this created a further hurdle for some patients in accessing therapy. ${ }^{17}$

A recent review of published evidence ${ }^{19}$, looked at a range of interventions to improve HCV treatment in people with opioid-use disorders, including engagement and educational programs, screening and treatment programs. Of the screening and treatment programs, a consistent finding was that a rapid, simplified work-up, and a single-location integrated treatment clinic were associated with favourable outcomes. ${ }^{19}$ Streamlining the work-up of cases might include coupling initial HCVantibody testing with tests for viraemia (i.e. HCV RNA). Similarly, determining whether a patient may have advanced liver disease can be established using the ALT to Platelet Ratio Index (APRI score), a measure calculated by utilising pathology values, rather than awaiting a specialised liver ultrasound, such as a Fibroscan, especially where the latter is not immediately available. In the future, using a point-of-care HCV test could simplify the process from diagnosis to treatment. Improving access to HCV treatment in primary care could reduce barriers that certain high-risk groups such as people who inject drugs face. Successful treatment programs have been conducted in a range of settings with strong success, including mobile teams, drug treatment services (e.g. combined with opioid-substitution programs or needle-syringe programs) and jails ${ }^{19}$, as well as nurse-led treatment initiatives. ${ }^{20,21}$

However, one limitation in building a primary-care based approach in South Western Sydney is that the majority of GPs in this study diagnosed HCV infection infrequently, with $90 \%$ of GPs detecting just a single case of HCV infection over the 5-month study period. This may be a factor contributing to the large proportion of GPs in our district who refer patients with HCV for specialist review, as a lack of experience in prescribing DAA therapies may be a barrier that medical practitioners encounter to starting antiviral treatment in their patients. Some evidence suggests that telementoring can play a useful role in this area, enabling GPs ready access to specialist support, for example via Project Echo. ${ }^{14}$

Other potential barriers to treatment include the likelihood that clinicians are managing several competing priorities where the patient is medically unwell or has comorbid medical conditions; $4 \%$ of patients in this study had a concurrent diagnosis of hepatocellular carcinoma (4\%) or died (5\%) shortly after their HCV notification. This study found another reason for not prescribing antiviral treatment included that $18 \%$ of patients were HCV RNAnegative, a rate in line with published literature ${ }^{22}$, and in this setting treatment is not indicated.

At a system level, there is also a strong argument for building more robust and proactive methods of critical result follow-up at the laboratory and PHU levels. This could involve the active follow-up of medical practitioners who have made a new HCV notification to ensure they have a patient recall process in place, and to offer practical support to assist a patient in accessing treatment. There is also a role for more GP training and public awareness campaigns regarding the importance of patients knowing their HCV status if they are in an atrisk group. Primary care databases and hospital-based electronic medical records potentially allow patients to be flagged who need recall for further assessment or testing (e.g. of HCV RNA).

\section{Study limitations}

Limitations of this study include the methodological variation in how data was collected in the GP and specialist groups, with phone interview predominantly used for GPs and email-based communication for specialists. Secondly, the data collection period was performed at an earlier stage for GP-detected versus 
specialist cases, which does not allow direct comparison of the two groups; however, the main finding that the majority of GPs in South Western Sydney prefer to refer on patients with a new HCV notification is not likely to change with more time. Also, we were unable to detail the reasons why a proportion of patients were lost to followup or comment on the proportion of patients treated with DAA therapy who achieved sustained virologic response in this study. The actual treatment rate could have been higher than that which was established here. The treatment details of those with a new HCV notification who were subsequently incarcerated or deported were not available, nor the treatment rate of those referred to specialists. The PHU process of recording, verifying a notification and removing duplicates was also not explicit or available to the study investigators. Of note, three false-positive notifications were made, suggesting the notification process has limitations in determining new cases of anti-HCV positivity in SWSLHD.

\section{Real-world implications}

By using PHU notification data, this study was able to determine the HCV treatment rates of those newly diagnosed with HCV infection (via a positive HCVantibody test) in a real-world setting. A large number of HCV-positive patients are living in the community who have not yet been diagnosed ${ }^{23}$, or linked into medical follow-up and treatment. Public health notification data represent a known cohort of HCV-antibody-positive patients who would ideally be contacted and treated if indicated. This study highlights the need for resources to be allocated to provide targeted, proactive follow-up of patients newly found to be HCV-antibody positive. These issues should be addressed in Australia's effort towards HCV elimination, in line with the World Health Organization goal of HCV elimination by $2030 .{ }^{12}$ Further research is required to refine models of care to enable patients to access antiviral treatment and to support GPs in managing $\mathrm{HCV}$ infection in primary care.

\section{Conclusion}

We found that $47 \%$ of patients newly diagnosed with HCV in this study time period and eligible for treatment were treated with antiviral therapy (excluding those referred by GPs to specialists). More than one-quarter of patients in this cohort were referred to a specialist, suggesting GPs in SWSLHD prefer to refer rather than treat HCV, which is understandable given the low rates of new HCV infection within their individual practices: most GPs in this study diagnosed one case of HCV in the 5-month study period. Almost one-third of patients eligible for antiviral treatment were lost to follow-up, and this is a great obstacle in the quest for HCV elimination. Future research could focus on the effectiveness of targeted interventions to link patients who are newly diagnosed with $\mathrm{HCV}$ into follow-up care.

\section{Peer review and provenance}

Externally peer reviewed, not commissioned.

\section{Competing interests}

ML has received consultancy payments from pharmaceutical companies Gilead Sciences and Bayer and speaking fees from MSD Australia, for work relating to liver disease including hepatitis C. The Liverpool Hospital Department of Gastroentrology and Hepatology received a $\$ 20000$ grant from AbbVie to provide patient resources related to HCV medications.

\section{Author contributions}

EO contributed to specialist data collection and was the lead author of the manuscript. MW and TL contributed to GP data collection and reviewed the manuscript, and ML supervised the study and methodology and reviewed the manuscipt.

\section{References}

1. The Kirby Institute. Hepatitis B and C in Australia: annual surveillance report supplement 2016. Sydney: The Kirby Institute, UNSW Sydney; 2016 [cited 2020 Apr 15]. Available from: kirby.unsw.edu.au/sites/default/files/kirby/ report/SERP_HepBandC-Annual-Surveillance-ReportSupp-2016.pdf

2. Gidding HF, Topp L, Middleton M, Robinson K, Hellard M, McCaughan $\mathrm{G}$, et al. The epidemiology of hepatitis C in Australia: notifications, treatment uptake and liver transplantations, 1997-2006. J Gastroenterol Hepatol. 2009;24(10):1648-54.

3. Australian Government Department of Health. Fifth national hepatitis C strategy, 2018-2022. Canberra: Commonwealth of Australia; 2018 [cited 2020 Apr 15]. Available from: www1.health.gov.au/internet/main/ publishing.nsf/Content/ohp-bbvs-1//\$File/Hep-C-Fifth-NatStrategy-2018-22.pdf

4. Burnet Institute and Kirby Institute. Australia's progress towards hepatitis C elimination: annual report 2019. Melbourne: Burnet Institute; 2019 [cited 2020 Apr 15]. Available from: kirby.unsw.edu.au/sites/default/files/kirby/ report/Australia\%27s-progress-on-hepatitis-C-elimination2019-report.pdf

5. de Kluyver R. Australia's notifiable disease status, 2013: annual report of the national notifiable diseases surveillance system. Canberra: Australian Government Department of Health; 2015 [cited 2020 Apr 15]. Available from: www1.health.gov.au/internet/main/ publishing.nsf/Content/cda-cdi3903-pdf-cnt.htm/\$FILE/ cdi3903i.pdf 
6. Hepatitis C Virus Infection Consensus Statement Working Group. Australian recommendations for the management of hepatitis $C$ virus infection: a consensus statement (September 2018). Melbourne: Gastroenterological Society of Australia; 2018 [cited 2020 Apr 15]. Available from: www.asid.net.au/documents/item/1208/

7. Westbrook RH, Dusheiko G. Natural history of hepatitis C. J Hepatol. 2014;61(1 Suppl):S58-68.

8. Puoti M, Foster GR, Wang S, Mutimer D, Gane E, Moreno C, et al. High SVR12 with 8-week and 12-week glecaprevir/pibrentasvir therapy: an integrated analysis of HCV genotype 1-6 patients without cirrhosis. J Hepatol. 2018; 69(2):293-300.

9. The Kirby Institute. Monitoring hepatitis $C$ treatment uptake in Australia (Issue 8). Sydney: The Kirby Institute, UNSW Sydney; 2017 [cited 2020 Apr 15]. Available from: kirby.unsw.edu.au/sites/default/files/kirby/report/ Monitoring-hep-C-treatment-uptake-in-Australia_Iss8DEC17.pdf

10. Belli LS, Perricone G, Adam R, Cortesi PA, Strazzabosco M, Facchetti R, et al. Impact of DAAs on liver transplantation: major effects on the evolution of indications and results. An ELITA study based on the ELTR registry. J Hepatology. 2018;69(4):810-7.

11. Hajarizadeh B, Grebely J, Matthews GV, Martinello M, Dore GJ. Uptake of direct-acting antiviral treatment for chronic hepatitis C in Australia. J Viral Hepat. 2018;25(6):640-8.

12. Global Hepatitis Program, Department of HIV/AIDS. Combating hepatitis $\mathrm{B}$ and $\mathrm{C}$ to reach elimination by 2030. Geneva: World Health Organisation; 2016 [cited 2020 Apr 15]. Available from: apps.who.int/iris/bitstream/ handle/10665/206453/WHO_HIV_2016.04_eng.pdf;jsessi onid=098682C4234F426BEB10DA4B7C1E37C7?sequen $\mathrm{ce}=1$

13. Scott N, Sacks-Davis R, Wade AJ, Stoove M, Pedrana A, Doyle JS, et al. Australia needs to increase testing to achieve hepatitis $\mathrm{C}$ elimination. Med J Aust. 2020;212(8):365-70.
14. Mohsen W, Chan P, Whelan M, Glass A, Mouton M, Yeung $E$, et al. Hepatitis $C$ treatment for difficult to access populations: can telementoring (as distinct from telemedicine) help? Intern Med J. 2018;49(3):351-7.

15. NSW Health. NSW Hepatitis B and C Strategies, 20142020: 2017 annual data report. Sydney: New South Wales Government; 2017 [cited 2020 Apr 15]. Available from: www.health.nsw.gov.au/hepatitis/Publications/2017annual-data-report.pdf

16. Robotin MC, Copland J, Tallis G, Coleman D, Giele C, Carter $\mathrm{L}$, et al. Surveillance for newly acquired hepatitis C in Australia. J Gastroenterol Hepatol. 2004;19:283-8.

17. Li V, Chen J. The burden of hepatitis $C$ in the injection drug user population the framework of stigma. J Glob Health. 2014

18. McGinley J, Schofield J, Garthwaite M, Sichi-Smith G, Laverty L, Peters SE. Hepatitis $C$ therapy delivered within and by a community addiction service: real life data shows SVR rates >90\%. J Hepatol. 2017;66(1):S512.

19. Rocero C, Ryan P, Littlewood R, Macias J, Ruiz J, Seijo P, et al. Practical steps to improve chronic hepatitis $\mathrm{C}$ treatment in people with opioid use disorder. Hepat Med. 2019;11:1-11.

20. Fragomeli V, Weltman M. Addressing viral hepatitis in the opiate substitution setting: an integrated nursing model of care. J Gastroenterol Hepatol. 2015;30(Suppl 2):6-11.

21. Pawlotsky J-M, Feld JJ, Zeuzem S, Hoofnagle JH. From non-A, non-B hepatitis to hepatitis $C$ virus cure. J Hepatol. 2015;62:S87-99.

22. Grebely J, Raffa JD, Lai C, Krajden M, Conway B, Tyndall MW. Factors associated with spontaneous clearance of hepatitis $\mathrm{C}$ among illicit drug users. Can J Gastroenterol. 2007;21(7):447-51.

23. Anderson EM, Mandeville RP, Hutchinson SJ, Cameron SO, Mills PR, Fox R, et al. Evaluation of a general practice based hepatitis $C$ virus screening intervention. Scott Med J. 2009;54(3):3-7.

\section{Copyright: (cc)}

(C) 2020 O'Brien et al. This article is licensed under the Creative Commons Attribution-NonCommercial-ShareAlike 4.0 International Licence, which allows others to redistribute, adapt and share this work non-commercially provided they attribute the work and any adapted version of it is distributed under the same Creative Commons licence terms. See: www.creativecommons.org/licenses/by-nc-sa/4.0/ 